\title{
A Lévy Risk Model with Ratcheting Dividend Strategy and Historic High-Related Stopping
}

\author{
Aili Zhang ${ }^{1}$ and Zhang Liu (D) $^{2,3}$ \\ ${ }^{1}$ School of Statistics and Mathematics, Nanjing Audit University, Nanjing 211815, China \\ ${ }^{2}$ School of Computer and Information Engineering, Jiangxi Agricultural University, Nanchang 330045, China \\ ${ }^{3}$ School of Mathematics and Statistics, Wuhan University, Wuhan 430072, China \\ Correspondence should be addressed to Zhang Liu; liuzhang1006@163.com
}

Received 15 January 2020; Accepted 14 February 2020; Published 26 March 2020

Guest Editor: Wenguang Yu

Copyright ( 92020 Aili Zhang and Zhang Liu. This is an open access article distributed under the Creative Commons Attribution License, which permits unrestricted use, distribution, and reproduction in any medium, provided the original work is properly cited.

\begin{abstract}
This paper focuses on the De Finetti's dividend problem for the spectrally negative Lévy risk process, where the dividend is deducted from the surplus process according to the racheting dividend strategy which was firstly introduced in Albrecher et al. (2018). A major feature of the racheting strategy lies in which the dividend rate never decreases. Unlike the conventional studies, the closed form expression for the expected, accumulated, and discounted dividend payments until the draw-down time (rather than the ruin time) is obtained in terms of the scale functions corresponding to the underlying Lévy process. The optimal barrier for the ratcheting strategy is also studied, where the dividend rate can be increased. Finally, two special cases, where the scale functions are explicitly known, i.e., the Brownian motion with drift and the compound Poisson model, are considered to illustrate the main result.
\end{abstract}

\section{Introduction}

During the first half of the 20th century, the actuaries concentrated on assessing the stability of an insurance company via the probability of ruin. In the seminal paper of De Finetti [1], the drawbacks of this approach was pointed out and an alternative approach was proposed, say, the approach of the expected discounted accumulated dividend payments, laying the foundations of De Finetti's dividend problem which has become a flourishing research topic now. In addition, the classical work of Miller and Modigliani [2] also claimed that the value of the firm can be equated with the discounted accumulated value of the dividend payments up to the infinite horizon. Roughly speaking, the classical De Finetti's dividend problem is to optimize the time and amount of dividends paid to the shareholders under certain criterion. In [1], where the surplus dynamics followed a discrete time random walk, De Finetti showed that a barrier dividend strategy is the optimal dividend strategy because it produces the maximum firm value of the company. From then on, the optimality of the barrier dividend strategy has been proved for various risk models under suitable assumptions, see, for instance, Loeffen [3], Loeffen and Renaud [4], Yin and Wang [5], Yuen and Yin [6], Wang and Zhou [7], Yu et al. [8], and the references therein.

Once the barrier dividend strategy with barrier $b$ is adopted by the controller of the company, all overflow part of the surplus above $b$ shall be paid out as dividends, i.e., the rate dividends paid out can be infinite. However, this requires that the controller has unlimited ability to control, which is often obviously not feasible nor realistic in real-life implementation. In addition, although the barrier dividend strategy commonly turns out to be the optimal one in many existing works, its optimality can easily vanish due to a different choice of the parameters of the underlying process. For example, it is seen from Loeffen [3] that the optimality of the barrier dividend strategy may fail when the assumption of a completely monotone density of the underlying process fails. Furthermore, a risk process imposed with a barrier dividend strategy has an ultimate ruin probability 1 (see, 
Gerber and Shiu [9]), a scenario far from being acceptable. Therefore, it is reasonable to restrict ourselves in those dividend strategies that modify the underlying surplus process moderately, or, in those dividend strategies with bounded dividend rates.

A known subclass of dividend strategies with bounded dividend rates is the threshold dividend strategy; dividends are paid out at a constant rate whenever the surplus is above the dividend threshold (say, $b$ ), whereas no dividend is paid when the surplus is below $b$. To the best of our knowledge, the threshold dividend strategy, which is also known as the refracting dividend strategy, was firstly introduced by Jeanblanc-Picqué and Shiryaev [10] and Asmussen and Taksar [11] for the diffusion process, and by Gerber and Shiu [12], for the Cremér-Lundberg risk process. Recently, the periodic threshold dividend strategy under a perturbed compound Poisson model was studied in Peng et al. [13] and Liu et al. [14]. For original ideas and analogues of the threshold dividend strategy, the readers are referred to Gerber and Shiu [9] and Avanzi et al. [15]. It needs to be mentioned that, for De Finetti's dividend problems, where the dividend strategies are bounded from above in the dividend rates, the optimal dividend strategy yielding the largest expected discounted accumulated dividends is in many scenarios the threshold dividend strategy, see Jeanblanc-Picqué and Shiryaev [10], Asmussen and Taksar [11], Gerber and Shiu [12], Avanzi et al. [15], and the references therein.

Note that the rate of paying dividends obeying a threshold dividend strategy may decrease to 0 once the surplus drops from levels above $b$ to levels below $b$. While, in practice, the practitioners are unwilling to accept a reduction in the rate of the dividend payment stream because a decrease in the rate of paying dividends may lead to negative psychological impacts on shareholders and the firm value of the company. Taking into account these considerations, Albrecher et al. [16] introduced a dividend strategy called ratcheting strategy, where the dividend rate would never decrease over time, but would increase once the underlying process hits some $b$ and stay at this higher level until the time of ruin. The authors derived the corresponding formulas for the resulting expected accumulated discounted dividend payments until ruin. We believe that the ratcheting strategy represents another subclass of constrained dividend strategies (other than the threshold dividend strategies) that is comparatively more acceptable in the community of practitioners.

However, one may not ignore the issue that the ratcheting dividend strategy inevitably increases the risk of bankruptcy compared to the threshold dividend strategy because the former entails larger amount of modification to the underlying process. To address this issue, in this paper the draw-down stopping (see (6) for its definition) will be introduced into the Lévy risk process with dividends deducted according to the ratcheting dividend strategy. In fact, the classical ruin time is a reduced version of the drawdown stopping, with the latter being more reserve involved and more efficient in assessing the stability of the insurance company taking on risks. In addition, one can expect positive surplus at the termination of the business if the draw-down stopping is adopted rather than the ruin time. Therefore, the draw-down stopping allows the insurer to achieve a balance between dividend distribution and solvency and hence represents a nice solution to the issue of large bankruptcy risk associated with the ratcheting dividend strategy.

Actually, as a remarkable progress in risk measurement and management, the draw-down time has recently gained a lot of attention in actuarial sciences and many ruin-based results were extended to draw-down versions. To name a few, Avram et al. [17] generalized the results of Albrecher and Thonhauser [18] by considering a linear draw-down stopping. Wang and Zhou [19] defined a draw-down reflected process which can be used to characterise the risk process with capital injections and solved several fluctuation identities. More recently, Wang and Zhou [20] introduced the concept of draw-down Parisian ruin time for a spectrally negative Lévy risk process and obtained the $k$ th moment of the discounted total dividends paid according to the barrier dividend strategy until the draw-down Parisian ruin time, which generalized a result of Czarna and Palmowski [21]. In addition, Wang and Zhou [7] solved a general draw-down version of De Finetti's optimal dividend problem. Wang and Zhang [22] studied the optimal loss-carry-forward tax problem with the general draw-down stopping. For application of the draw-down time in risk theory, we are referred to Wang and Ming [23], Wang et al. [24], Chen et al. [25], Avram et al. [17], Ruan et al. [26], Yu et al. [27], and Zhang et al. [28].

In this paper, motivated by Albrecher et al. [16], Wang and Zhou [7], Wang and Zhou [20], and Wang and Zhang [22], we are to introduce the general draw-down time into the Lévy risk process with dividends deducted according to the racheting dividend strategy and to generalize the ruininvolved results of Albrecher et al. [16] to the general drawdown-based version. The expressions of the expected accumulated discounted dividend payments until the general draw-down time are obtained. The optimal surplus level where the dividend rate can be increased is also studied. All results are expressed in terms of the scale functions. Finally, two special cases where the scale functions are explicitly known, i.e., the Brownian motion with drift and the compound Poisson model with exponential jumps, are considered. The contribution of this paper lies in which our results shall serve as a nice solution to the concern of large bankruptcy risk associated with the ratcheting dividend strategy, hence achieve a balance between dividend distribution and solvency. It should be mentioned that the results in Albrecher et al. [16] can be recovered when the drawdown functions are specified to be 0 .

The rest of this paper is organized as follows. In Section 2, we review the spectrally negative Lévy processes, the associated scale functions, the draw-down time, and existing results of the exit problems for spectrally negative Lévy processes involving the general draw-down time. In Section 3 , we derive the formula for the expected accumulated discounted dividend payments until draw-down time according the ratcheting dividend strategy and obtain a 
criterion for the optimal ratcheting barrier. Section 4 studies two special spectrally negative Lévy processes and obtains the corresponding explicit expressions for the expected discounted dividend payments.

\section{Problem Formulation}

Let $Y=\left\{Y_{t} ; t \geq 0\right\}$ be a spectrally negative Lévy process defined on a filtered probability space $\left(\Omega,\left\{\mathscr{F}_{t} ; t \geq 0\right\}, \mathbb{P}\right)$ with the natural filtration $\left\{\mathscr{F}_{t} ; t \geq 0\right\}$. Denote by $\mathbb{P}_{x}$ the conditional probability given $Y_{0}=x$ and by $\mathscr{E}_{x}$ the associated conditional expectation. For notational convenience, we write $\mathbb{P}$ and $\mathscr{E}$ in place of $\mathbb{P}_{0}$ and $\mathscr{E}_{0}$, respectively. In this paper, the surplus process in the absence of dividends is expressed by $Y=\left\{Y_{t}\right.$; $t \geq 0\}$. We shall assume that the company will pay dividends to its shareholders according to the ratcheting strategy introduced by Albrecher et al. [16]. Roughly speaking, dividends are paid constantly at rate $c_{1} \geq 0$, and in certain periods at an increased rate $c_{1}+c_{2}$ with $c_{2}>0$. To characterise the modified surplus processes, we introduce

$$
\begin{aligned}
& X_{t}=Y_{t}-c_{1} t, \\
& \tilde{X}_{t}=Y_{t}-\left(c_{1}+c_{2}\right) t,
\end{aligned}
$$

for all $t \geq 0$. It should be mentioned that they are still spectrally negative Lévy processes.

The Laplace exponent of $X$ is defined by

$$
\psi(\theta):=\log \mathscr{E}_{x}\left[e^{\theta\left(X_{1}-x\right)}\right], \quad \theta \geq 0,
$$

which is strictly convex and infinitely differentiable.

Scale functions play a key role in analyzing spectrally negative Lévy processes. We now recall the definitions of the scale functions $W_{q}(x)$ and $Z_{q}(x)$ corresponding to $X$. For each $q \geq 0, W_{q}:[0, \infty) \longrightarrow[0, \infty)$ is the unique strictly increasing and continuous function with the Laplace transform:

$$
\int_{0}^{\infty} e^{-\lambda x} \mathbb{W}_{q}(x) \mathrm{d} x=\frac{1}{\psi(\lambda)-q}, \quad \lambda>\Phi_{q},
$$

where $\Phi_{q}$ is the largest solution of the equation $\psi(\lambda)=q$ (there are at most two). For convenience, we extend the domain of $W_{q}(x)$ to the whole real line by setting $W_{q}(x)=0$ for $x<0$. Associated to the function $W_{q}$, the function $Z_{q}$ is defined by

$$
Z_{q}(x)=1+q \int_{0}^{x} \mathbb{W}_{q}(z) \mathrm{d} z, \quad x \geq 0 .
$$

Throughout the paper, we assume $W_{q}(x)$ is differentiable. It is known that when $X$ has sample paths of unbounded variation or when $X$ has sample paths of bounded variation and the Lévy measure has no atoms, the scale function $W_{q}(x)$ is continuously differentiable over $(0, \infty)$. The interested readers are referred Kuznetsov et al. [29] for more detailed discussions on the smoothness of scale functions. In addition, we define $\mathbb{W}_{q}(x)$ and $\mathbb{Z}_{q}(x)$ analogously for $\left\{\widetilde{X}_{t} ; t \geq 0\right\}$.

Let $\bar{X}_{t}=\sup _{0 \leq s \leq t} X_{s}$ denote the running maximum process for $X$. A measurable function $\xi$ defined on $\mathbb{R}$ is called a general draw-down function if $\xi(x)<x$ for all $x \in \mathbb{R}$. In the following, we define the first up-crossing time of the given process $X$ for a fixed $b \geq 0$ and the general draw-down time of $X$ with the draw-down function $\xi$, respectively, as

$$
\begin{aligned}
\tau_{b}^{+} & :=\inf \left\{t \geq 0, X_{t}>b\right\}, \\
\tau_{\xi} & :=\inf \left\{t \geq 0, X_{t}<\xi\left(\bar{X}_{t}\right)\right\},
\end{aligned}
$$

with the convention that $\inf \varnothing=\infty$.

Remark 1 . When $\xi \equiv 0, \tau_{\xi}$ reduces to the classical ruin time. When the draw-down function is linear, i.e., $\xi\left(\bar{X}_{t}\right)=k \bar{X}_{t}-$ $d$ for $k<1, X_{t}<\xi\left(\bar{X}_{t}\right)$ is identical to $k \bar{X}_{t}-X_{t}>d$; hence, $\tau_{\xi}$ refers for the first time the surplus process $X_{t}$ drops more than $d$ units below $k \bar{X}_{t}$. Examples of nonlinear forms of $\xi$ can be found in Remark 2 of Avram et al. [17].

Remark 2. By definition, the draw-down time is the first time when a drop of the surplus process from the running maximum exceeds a certain surplus-related level. This nature of the draw-down time assures its feasibility of applying it in measuring and managing extreme risks. In practice, people can select the draw-down functions according to management needs and attitudes toward risks, for example, risk-averse insurer may choose larger draw-down function, vice versa. In addition, one can adjust the draw-down function so that the surplus remains positive at the drawdown time with positive probability, see Wang and Zhang [22] and Wang and Zhou [7]. The insurer may deem $\xi\left(\bar{X}_{t}\right)$ as a warning line (over time) for taking actions to avoid even worse situations, see Wang et al. [24]. Moreover, the drawdown function can also be chosen to be negative valued, in this case the draw-down time is also called the absolute ruin time, see Wang and Zhang [22] and Landriault et al. [30].

The following solution for the draw-down-based twoside exit problem is found in Proposition 3.1 of Li et al. [31] and Wang and Zhou [7], which will be used later on.

Lemma 1 (two-side exit problem involving draw-down). For $b \in[0, \infty)$ and $x \in[0, b)$, we have

$$
\begin{aligned}
\mathbb{E}_{x}\left(e^{-q \tau_{b}^{+}} 1_{\left\{\tau_{b}^{+}<\tau_{\xi}\right\}}\right) & =\exp \left(-\int_{x}^{b} \frac{\mathbb{W}_{q}^{\prime}(\bar{\xi}(z))}{\mathbb{W}_{q}(\bar{\xi}(z))} \mathrm{d} z\right), \\
\mathbb{E}_{x}\left(e^{-q \tau_{\xi}} 1_{\left\{\tau_{\xi}<\tau_{b}^{+}\right\}}\right)= & \int_{x}^{b} \exp \left(-\int_{x}^{z} \frac{\mathbb{W}_{q}^{\prime}(\bar{\xi}(w))}{\mathbb{W}_{q}(\bar{\xi}(w))} \mathrm{d} w\right) \\
& \times\left(\frac{\mathbb{W}_{q}^{\prime}(\bar{\xi}(z))}{\mathbb{W}_{q}(\bar{\xi}(z))} Z_{q}(\bar{\xi}(z))\right. \\
& \left.-q \mathbb{W}_{q}(\bar{\xi}(z))\right) \mathrm{d} z,
\end{aligned}
$$

where $\bar{\xi}(z):=z-\xi(z)$.

Remark 3. Letting $b \longrightarrow \infty$ in (8), we can obtain the Laplace transform of the general draw-down time of the Lévy risk process as follows: 


$$
\begin{aligned}
\mathbb{E}_{x}\left(e^{-q \tau_{\xi}}\right)= & \int_{x}^{\infty} \exp \left(-\int_{x}^{z} \frac{\mathbb{W}_{q}^{\prime}(\bar{\xi}(w))}{\mathbb{W}_{q}(\bar{\xi}(w))} \mathrm{d} w\right) \\
& \cdot\left(\frac{\mathbb{W}_{q}^{\prime}(\bar{\xi}(z))}{\mathbb{W}_{q}(\bar{\xi}(z))} Z_{q}(\bar{\xi}(z))-q \mathbb{W}_{q}(\bar{\xi}(z))\right) \mathrm{d} z .
\end{aligned}
$$

\section{Problem Presentation and the Main Results}

In this section, we consider the racheting dividend strategy with a general draw-down time and obtain our main results. Under the racheting strategy with barrier level $b$, dividends are paid at a fixed constant rate $c_{1} \geq 0$ until the first time the surplus process hits a barrier $b$, and from then the dividend rate is increased (racheted) to $c_{1}+c_{2}$ for a fixed constant $c_{2}>0$. The cumulative dividends paid out up to time $t$ is represented by $D_{t}=\int_{0}^{t}\left(c_{1}+c_{2} 1_{\left\{M_{s} \geq b\right\}}\right) \mathrm{d} s$, where $M_{t}:=\sup _{0 \leq s \leq t} X_{s}$. The surplus process after the deduction of dividends is given by

$$
U_{t}=Y_{t}-D_{t}=X_{t}-\int_{0}^{t} c_{2} 1_{\left\{M_{s} \geq b\right\}} \mathrm{d} s
$$

In order to balance the dividend optimization and solvency (see also paragraph 5 of Section 1), we consider the value function of the dividend strategy by the expected value of the accumulated discounted dividend payments until the drawdown time, instead of the ruin time. The draw-down time when the dividend payments are taken into account is defined by

$$
\tau_{\xi}^{u}:=\inf \left\{t \geq 0, U_{t}<\xi\left(\bar{U}_{t}\right)\right\}
$$

where $\bar{U}_{t}=\sup _{0 \leq s \leq t} U_{s}$. The expected value of the accumulated discounted dividend payments until the draw-down time under the ratcheting strategy can be expressed by

$$
V_{\xi}(x)=\mathbb{E}_{x}\left(\int_{0}^{\tau_{\xi}^{u}} e^{-q t}\left(c_{1}+c_{2} 1_{\left\{M_{t} \geq b\right\}}\right) \mathrm{d} t\right),
$$

where $q$ is the force of interest for valuation. In this paper, our goal is to obtain the expression of the expected value of the accumulated discounted dividend payments until the draw-down time under the ratcheting strategy and find the optimal level $b$ for the fixed $c_{1}$ and $c_{2}$.

The following Theorem 1 gives the expression of $V_{\xi}$ in terms of the scale functions of $X$ and $\widetilde{X}$.

Theorem 1. The expected value of the accumulated discounted dividend payments until the draw-down time under the ratcheting strategy for the modified surplus process is given as follows.

(1) For $x \in[b, \infty)$, we have

$$
\begin{aligned}
V_{\xi}(x)= & \frac{c_{1}+c_{2}}{q}\left[1-\int_{x}^{\infty} \exp \left(-\int_{x}^{z} \frac{\mathbb{W}_{q}^{\prime}(\bar{\xi}(w))}{\mathbb{W}_{q}(\bar{\xi}(w))} \mathrm{d} w\right)\right. \\
& \left.\left.\times\left(\frac{\mathbb{W}_{q}^{\prime}(\bar{\xi}(z))}{\mathbb{W}_{q}(\bar{\xi}(z))} \mathbb{Z}_{q}(\bar{\xi}(z))-q \mathbb{W}_{q}(\bar{\xi}(z))\right) \mathrm{d} z\right)\right] .
\end{aligned}
$$

(2) For $x \in[0, b)$, we have

$$
\begin{aligned}
V_{\xi}(x)= & \frac{c_{1}}{q}-\frac{c_{1}}{q} \int_{x}^{b} \exp \left(-\int_{x}^{z} \frac{\mathbb{W}_{q}^{\prime}(\bar{\xi}(w))}{\mathbb{W}_{q}(\bar{\xi}(w))} \mathrm{d} w\right) \\
& \cdot\left(\frac{\mathbb{W}_{q}^{\prime}(\bar{\xi}(z))}{\mathbb{W}_{q}(\bar{\xi}(z))} Z_{q}(\bar{\xi}(z))-q \mathbb{W}_{q}(\bar{\xi}(z))\right) \mathrm{d} z \\
& +\exp \left(-\int_{x}^{b} \frac{\mathbb{W}_{q}^{\prime}(\bar{\xi}(z))}{\mathbb{W}_{q}(\bar{\xi}(z))} \mathrm{d} z\right)\left(V_{\xi}(b)-\frac{c_{1}}{q}\right) .
\end{aligned}
$$

Proof.

(1) For $x \in[b, \infty)$, the dividends are paid out at the rate $c_{1}+c_{2}$ from the beginning. In this case, the modified surplus process is given by $U_{t}=Y_{t}-$ $\int_{0}^{t}\left(c_{1}+c_{2}\right) \mathrm{d} s=\widetilde{X}_{t}$, and

$$
\begin{aligned}
V_{\xi}(x) & =\left(c_{1}+c_{2}\right) \mathbb{E}_{x}\left(\int_{0}^{\tau_{\xi}^{u}} e^{-q t} \mathrm{~d} t\right) \\
& =\frac{c_{1}+c_{2}}{q} \mathbb{E}_{x}\left(1-e^{-\tilde{q}_{\xi}}\right),
\end{aligned}
$$

where $\quad \tilde{\tau}_{\xi}:=\inf \left\{t \geq 0, \widetilde{X}_{t}<\xi\left(\widetilde{X}_{t}\right)\right\}$. Under the framework of $\tilde{X}$, using Remark 3, we can get (13) immediately.

(2) For $x \in[0, b)$, we should consider whether the process will reach $b$ before the first time of dropping across the level of the draw-down function of the running maximum. If the process touches the barrier $b$ first, i.e., $\tau_{b}^{+}<\tau_{\xi}^{u}$, the strong Markov property will be used at that point in time $\tau_{b}^{+}$, and from which the dividend rate is ratcheted to $c_{1}+c_{2}$. Hence,

$$
\begin{aligned}
V_{\xi}(x)= & c_{1} \mathbb{E}_{x}\left(1_{\left\{\tau_{\xi}^{u}<\tau_{b}^{+}\right\}} \int_{0}^{\tau_{\xi}^{u}} e^{-q t} \mathrm{~d} t\right) \\
& +\left[c_{1} \mathbb{E}_{x}\left(1_{\left\{\tau_{b}^{+}<\tau_{\xi}^{u}\right\}} \int_{0}^{\tau_{b}^{+}} e^{-q t} \mathrm{~d} t\right)\right. \\
& \left.+\mathbb{E}_{x}\left(e^{-q \tau_{b}^{+}} 1_{\left\{\tau_{b}^{+}<\tau_{\xi}^{u}\right\}}\right) V_{\xi}(b)\right] \\
= & \frac{c_{1}}{q}-\frac{c_{1}}{q} \mathbb{E}_{x}\left(e^{-q \tau_{\xi}^{u}} 1_{\left\{\tau_{\xi}^{u}<\tau_{b}^{+}\right\}}\right) \\
& -\left[\frac{c_{1}}{q} \mathbb{E}_{x}\left(e^{-q \tau_{b}^{+}} 1_{\left\{\tau_{b}^{+}<\tau_{\xi}^{u}\right\}}\right)\right. \\
& \left.-\mathbb{E}_{x}\left(e^{-q \tau_{b}^{+}} 1_{\left\{\tau_{b}^{+}<\tau_{\xi}^{u}\right\}}\right) V_{\xi}(b)\right] \\
= & \frac{c_{1}}{q}-\frac{c_{1}}{q} \mathbb{E}_{x}\left(e^{-q \tau_{\xi}} 1_{\left\{\tau_{\xi}<\tau_{b}^{+}\right\}}\right)-\mathbb{E}_{x}\left(e^{-q \tau_{b}^{+}} 1_{\left\{\tau_{b}^{+}<\tau_{\xi}\right\}}\right) \\
& \left(V_{\xi}(b)-\frac{c_{1}}{q}\right),
\end{aligned}
$$


where $V_{\xi}(b)$ is given by (13). According to the formulations of the two-side exit problem involving dawn-down time in Lemma 1, we can obtain (14).
The following corollaries will present the expressions of $V_{\xi}(x)$ with $\xi(x)=k x-a$.

Corollary 1. For $k<1$ and $a>0$, we have, for $x \in[b, \infty)$,

$$
\begin{aligned}
V_{k x-a}(x)= & \frac{c_{1}+c_{2}}{q}\left(1-\mathbb{Z}_{q}((1-k) x+a)+q k\left(\mathbb{W}_{q}((1-k) x+a)\right)^{1 /(1-k)}\right. \\
& \left.\times \int_{x}^{\infty}\left(\mathbb{W}_{q}((1-k) z+a)\right)^{-k /(1-k)} \mathrm{d} z\right),
\end{aligned}
$$

and for $x \in[0, b)$,

$$
\begin{aligned}
V_{k x-a}(x)= & \frac{c_{1}}{q}\left(1-Z_{q}((1-k) x+a)+Z_{q}((1-k) b+a)\left(\frac{\mathbb{W}_{q}((1-k) x+a)}{\mathbb{W}_{q}((1-k) b+a)}\right)^{1 /(1-k)}+q k\left(\mathbb{W}_{q}((1-k) x+a)\right)^{1 /(1-k)}\right. \\
& \left.\cdot \int_{x}^{b}\left(\mathbb{W}_{q}((1-k) z+a)\right)^{1 /(1-k)} \mathrm{d} z\right)+\left(\frac{\mathbb{W}_{q}((1-k) x+a)}{\mathbb{W}_{q}((1-k) b+a)}\right)^{1 /(1-k)}\left(V_{k x-a}(b)-\frac{c_{1}}{q}\right) .
\end{aligned}
$$

Corollary 2. For $k=1$ and $a>0$, we have, for $x \in[b, \infty)$,

$$
V_{\xi}(x)=\frac{c_{1}+c_{2}}{q}\left(1-\mathbb{Z}_{q}(a)+q \frac{1}{\mathbb{W}_{q}^{\prime}(a)}\right),
$$

and for $x \in[0, b)$,

$$
\begin{aligned}
V_{\xi}(x)= & \frac{c_{1}}{q}\left(1-Z_{q}(a)+q \frac{1}{\mathbb{W}_{q}^{\prime}(a)}\right) \\
& -\left[\frac{c_{1}}{q}\left(1-Z_{q}(a)+q \frac{1}{\mathbb{W}_{q}^{\prime}(a)}\right)\right. \\
& \left.-\frac{c_{1}+c_{2}}{q}\left(1-\mathbb{Z}_{q}(a)+q \frac{1}{\mathbb{W}_{q}^{\prime}(a)}\right)\right] \\
& \exp \left(-\frac{\mathbb{W}_{q}^{\prime}(a)}{\mathbb{W}_{q}(a)}(b-x)\right) .
\end{aligned}
$$

Remark 4. If $\xi(x) \equiv 0$, the draw-down time $\tau_{\xi}^{u}=\inf \{t \geq$ $\left.0, U_{t}<0\right\}$ is the classical ruin time of $U_{t}$, and (13) and (14) reduce to that of Theorem 2.1 of Albrecher et al. [16].

Denote the right-hand side of (13) and (14) by $V_{\xi}^{+}(x)$ and $V_{\xi}^{-}(x, b)$, respectively. For fixed $x \in[0, \infty)$, define a function $V_{\xi}(x, b)$ of $b$ and the largest global maximum point $b^{*}$ of $V_{\xi}(x, b)$ as

$$
\begin{gathered}
V_{\xi}(x, b):=V_{\xi}^{+}(x) 1_{[0, x]}(b)+V_{\xi}^{-}(x, b) 1_{(x, \infty)}(b), \\
b^{*}:=\sup \left\{b_{0} \geq 0 \mid V_{\xi}\left(x, b_{0}\right) \geq V_{\xi}(x, b), \forall b \geq 0\right\} .
\end{gathered}
$$

Hence, $b^{*}$ is the optimal barrier level that maximizes the expectation of the accumulated discounted dividends until the draw-down time. For the purpose of simplification, we shall define some auxiliary functions as

$$
\begin{aligned}
\iota_{\xi}(x) & =\frac{\mathbb{W}_{q}^{\prime}(\bar{\xi}(x))}{\mathbb{W}_{q}(\bar{\xi}(x))} Z_{q}(\bar{\xi}(x))-q \mathbb{W}_{q}(\bar{\xi}(x)), \\
\varrho_{\xi}(x) & =\frac{\mathbb{W}_{q}^{\prime}(\bar{\xi}(x))}{\mathbb{W}_{q}(\bar{\xi}(x))} .
\end{aligned}
$$

Similarly,

$$
\begin{aligned}
\tilde{\iota}_{\xi}(x) & =\frac{\mathbb{W}_{q}^{\prime}(\bar{\xi}(x))}{\mathbb{W}_{q}(\bar{\xi}(x))} \mathbb{Z}_{q}(\bar{\xi}(x))-q \mathbb{W}_{q}(\bar{\xi}(x)), \\
\widetilde{\varrho}_{\xi}(x) & =\frac{\mathbb{W}_{q}^{\prime}(\bar{\xi}(x))}{\mathbb{W}_{q}(\bar{\xi}(x))} .
\end{aligned}
$$

Proposition 1. Let $b^{*}$ be given by (21) with fixed $c_{1}, c_{2}>0$ and $x \geq 0$. If $b^{*} \in(x, \infty)$, then $b^{*}$ must be solution of

$$
\begin{aligned}
c_{1}\left[\iota_{\xi}(b)+\varrho_{\xi}(b)\left(\frac{q}{c_{1}} V_{\xi}(b)-1\right)\right]= & \left(c_{1}+c_{2}\right)\left[\widetilde{\iota}_{\xi}(b)+\widetilde{\varrho}_{\xi}(b)\right. \\
& \left.\left(\frac{q}{c_{1}+c_{2}} V_{\xi}(b)-1\right)\right] .
\end{aligned}
$$

Proof. For fixed $x \in[0, \infty)$, it is seen by $(21)$ that $V_{\xi}(x, b)$ is flat over $[0, x]$, i.e., $V_{\xi}(x, b) \equiv V_{\xi}^{+}(x)$ for all $b \in[0, x]$; hence, the optimal threshold level $b^{*} \in[x, \infty]$. 
If $b^{*} \in(x, \infty)$, then by $(21)$, one knows that $b^{*}$ must be a zero of the equation $(\partial / \partial b) V_{\xi}^{-}(x, b)=0$, i.e.,

$$
\begin{aligned}
0= & \frac{\partial}{\partial b} V_{\xi}^{-}(x, b)=\exp \left(-\int_{x}^{b} \frac{\mathbb{W}_{q}^{\prime}(\bar{\xi}(w))}{\mathbb{W}_{q}(\bar{\xi}(w))} \mathrm{d} w\right) \\
& \cdot\left[-\frac{c_{1}}{q}\left(\frac{\mathbb{W}_{q}^{\prime}(\bar{\xi}(b))}{\mathbb{W}_{q}(\bar{\xi}(b))} Z_{q}(\bar{\xi}(b))-q \mathbb{W}_{q}(\bar{\xi}(b))\right)-\frac{\mathbb{W}_{q}^{\prime}(\bar{\xi}(b))}{\mathbb{W}_{q}(\bar{\xi}(b))}\left(V_{\xi}^{+}(b)-\frac{c_{1}}{q}\right)+\frac{c_{1}+c_{2}}{q}\left(\frac{\mathbb{W}_{q}^{\prime}(\bar{\xi}(b))}{\mathbb{W}_{q}(\bar{\xi}(b))} \mathbb{Z}_{q}(\bar{\xi}(b))-q \mathbb{W}_{q}(\bar{\xi}(b))\right)\right. \\
& \left.-\frac{c_{1}+c_{2}}{q} \frac{\mathbb{W}_{q}^{\prime}(\bar{\xi}(b))}{\mathbb{W}_{q}(\bar{\xi}(b))} \int_{b}^{\infty} \exp \left(-\int_{b}^{z} \frac{\mathbb{W}_{q}^{\prime}(\bar{\xi}(w))}{\mathbb{W}_{q}(\bar{\xi}(w))} \mathrm{d} w\right) \times\left(\frac{\mathbb{W}_{q}^{\prime}(\bar{\xi}(z))}{\mathbb{W}_{q}(\bar{\xi}(z))} \mathbb{Z}_{q}(\bar{\xi}(z))-q \mathbb{W}_{q}(\bar{\xi}(z))\right) \mathrm{d} z\right] \\
= & \exp \left(-\int_{x}^{b} \varrho_{\xi}(w) \mathrm{d} w\right)\left[-\frac{c_{1}}{q} l_{\xi}(b)-\varrho_{\xi}(b)\left(V_{\xi}(b)-\frac{c_{1}}{q}\right)+\frac{c_{1}+c_{2}}{q} \widetilde{\tau}_{\xi}(b)+\frac{c_{1}+c_{2}}{q} \widetilde{\varrho}_{\xi}(b)\left(\frac{q}{c_{1}+c_{2}} V_{\xi}(b)-1\right)\right] .
\end{aligned}
$$

The proof is complete.

The following proposition characterizes the optimal barrier level $b$ from the view of smoothness.
Proposition 2. In the ratcheting dividend problem, for the fixed $c_{1}, c_{2}>0$, the optimal barrier $b^{*} \in(x, \infty)$ coincides with the one which makes the $V_{\xi}(x)$ continuously differentiable.

Proof. For $x \in[0, b)$, we have

$$
\begin{aligned}
\frac{\partial}{\partial x} V_{\xi}(x)= & \frac{c_{1}}{q}\left(\frac{\mathbb{W}_{q}^{\prime}(\bar{\xi}(x))}{\mathbb{W}_{q}(\bar{\xi}(x))} Z_{q}(\bar{\xi}(x))-q \mathbb{W}_{q}(\bar{\xi}(x))\right) \\
& +\exp \left(-\int_{x}^{b} \frac{\mathbb{W}_{q}^{\prime}(\bar{\xi}(w))}{\mathbb{W}_{q}(\bar{\xi}(w))} \mathrm{d} w\right) \frac{\mathbb{W}_{q}^{\prime}(\bar{\xi}(x))}{\mathbb{W}_{q}(\bar{\xi}(x))}\left(V_{\xi}(b)-\frac{c_{1}}{q}\right)-\frac{c_{1}}{q} \frac{\mathbb{W}_{q}^{\prime}(\bar{\xi}(x))}{\mathbb{W}_{q}(\bar{\xi}(x))} \int_{x}^{b} \exp \left(-\int_{x}^{z} \frac{\mathbb{W}_{q}^{\prime}(\bar{\xi}(w))}{\mathbb{W}_{q}(\bar{\xi}(w))} \mathrm{d} w\right) \\
& \times\left(\frac{\mathbb{W}_{q}^{\prime}(\bar{\xi}(z))}{\mathbb{W}_{q}(\bar{\xi}(z))} Z_{q}(\bar{\xi}(z))-q \mathbb{W}_{q}(\bar{\xi}(z))\right) \mathrm{d} z \\
= & \frac{c_{1}}{q} l_{\xi}(x)+\exp \left(-\int_{x}^{b} \varrho_{\xi}(x) \mathrm{d} w\right) \varrho_{\xi}(x)\left(V_{\xi}(b)-\frac{c_{1}}{q}\right) \\
& -\frac{c_{1}}{q} \varrho_{\xi}(x) \int_{x}^{b} \exp \left(-\int_{x}^{z} \varrho_{\xi}(w) \mathrm{d} w\right) \iota_{\xi}(z) \mathrm{d} z,
\end{aligned}
$$

and for $x \in[b, \infty)$, we can obtain

$$
\begin{aligned}
\frac{\partial}{\partial x} V_{\xi}(x)= & -\frac{c_{1}+c_{2}}{q} \frac{\mathbb{W}_{q}^{\prime}(\bar{\xi}(x))}{\mathbb{W}_{q}(\bar{\xi}(x))} \int_{x}^{\infty} \exp \left(-\int_{x}^{z} \frac{\mathbb{W}_{q}^{\prime}(\bar{\xi}(w))}{\mathbb{W}_{q}(\bar{\xi}(w))} \mathrm{d} w\right) \times\left(\frac{\mathbb{W}_{q}^{\prime}(\bar{\xi}(z))}{\mathbb{W}_{q}(\bar{\xi}(z))} \mathbb{Z}_{q}(\bar{\xi}(z))-q \mathbb{W}_{q}(\bar{\xi}(z))\right) \mathrm{d} z \\
& +\frac{c_{1}+c_{2}}{q}\left(\frac{\mathbb{W}_{q}^{\prime}(\bar{\xi}(x))}{\mathbb{W}_{q}(\bar{\xi}(x))} \mathbb{Z}_{q}(\bar{\xi}(x))-q \mathbb{W}_{q}(\bar{\xi}(x))\right) \\
= & -\frac{c_{1}+c_{2}}{q} \widetilde{\varrho}_{\xi}(x) \int_{x}^{\infty} \exp \left(-\int_{x}^{z} \widetilde{\varrho}_{\xi}(w) \mathrm{d} w\right) \tilde{l}_{\xi}(z) \mathrm{d} z+\frac{c_{1}+c_{2}}{q} \widetilde{\iota}_{\xi}(x) .
\end{aligned}
$$


Letting $x \uparrow b$ in (28) and $x \downarrow b$ in (29), respectively, gives rise to

$$
\begin{gathered}
V_{\xi}^{\prime}(b-)=\frac{c_{1}}{q} l_{\xi}(b)+\varrho_{\xi}(b)\left(V_{\xi}(b)-\frac{c_{1}}{q}\right), \\
V_{\xi}^{\prime}(b+)=-\frac{c_{1}+c_{2}}{q} \widetilde{\varrho}_{\xi}(b) \int_{b}^{\infty} \exp \left(-\int_{b}^{z} \widetilde{\varrho}_{\xi}(w) \mathrm{d} w\right) \widetilde{\iota}_{\xi}(z) \mathrm{d} z+\frac{c_{1}+c_{2}}{q} \widetilde{\iota}_{\xi}(b) .
\end{gathered}
$$

In addition, applying the auxiliary functions, (13) can be expressed as

$$
\int_{b}^{\infty} \exp \left(-\int_{b}^{z} \tilde{\varrho}_{\xi}(w) \mathrm{d} w\right) \tilde{l}_{\xi}(z) \mathrm{d} z=1-\frac{q}{c_{1}+c_{2}} V_{\xi}(b) .
$$

Substitute (32) into (31) and then combine the yielding equation with (30), one can find that $V_{\xi}^{\prime}(b-)=V_{\xi}^{\prime}(b+)$ coincides with (26).

\section{Special Cases}

In this section, we shall examine two special cases of our model, i.e., the Brownian motion with drift and the compound Poison process. Explicit results of the expected accumulated discounted dividend payments until the general draw-down time are obtained.

Example 1. Let $Y_{t}=x+\mu t+\sigma B_{t}, t \geq 0$ with $\mu>0, \sigma>0$, and $\left\{B_{t} ; t \geq 0\right\}$ being a standard Brownian motion. In this case, the scale functions of $X_{t}$ and $\widetilde{X}_{t}$ are given, respectively, by

$$
\begin{aligned}
& \mathbb{W}_{q}(x)=\kappa\left(e^{\theta_{1} x}-e^{\theta_{2} x}\right), \\
& \mathbb{W}_{q}(x)=\widetilde{\kappa}\left(e^{\tilde{\theta}_{1} x}-e^{\tilde{\theta}_{2} x}\right),
\end{aligned}
$$

where

$$
\begin{aligned}
& \kappa=\left(\left(\mu-c_{1}\right)^{2}+2 \sigma^{2} q\right)^{-(1 / 2)}, \\
& \theta_{1}=\frac{c_{1}-\mu+\sqrt{\left(\mu-c_{1}\right)^{2}+2 \sigma^{2} q}}{\sigma^{2}}, \\
& \theta_{2}=\frac{c_{1}-\mu-\sqrt{\left(\mu-c_{1}\right)^{2}+2 \sigma^{2} q}}{\sigma^{2}}, \\
& \tilde{\kappa}^{2}=\left(\left(\mu-c_{1}-c_{2}\right)^{2}+2 \sigma^{2} q\right)^{-(1 / 2)}, \\
& \tilde{\theta}_{1}=\frac{\left(c_{1}+c_{2}\right)-\mu+\sqrt{\left(\mu-\left(c_{1}+c_{2}\right)\right)^{2}+2 \sigma^{2} q}}{\sigma^{2}}, \\
& \tilde{\theta}_{2}=\frac{\left(c_{1}+c_{2}\right)-\mu-\sqrt{\left(\mu-\left(c_{1}+c_{2}\right)\right)^{2}+2 \sigma^{2} q}}{\sigma^{2}} .
\end{aligned}
$$

They are well-known results (see Wang and Zhang [22]).

For $x, q \geq 0$, by some algebraic manipulations, we have

$$
\begin{aligned}
& Z_{q}(x)=\frac{q}{\theta_{1}} \mathbb{W}_{q}(x)+e^{\theta_{2} x}, \\
& \mathbb{Z}_{q}(x)=\frac{q}{\tilde{\theta}_{1}} \mathbb{W}_{q}(x)+e^{\tilde{\theta}_{2} x} .
\end{aligned}
$$

By (33) and (35), we can verify that 


$$
\begin{aligned}
\varrho_{\xi}(x)= & \frac{\mathbb{W}_{q}^{\prime}(\bar{\xi}(x))}{\mathbb{W}_{q}(\bar{\xi}(x))}=\frac{\theta_{1} e^{\theta_{1} \bar{\xi}(x)}-\theta_{2} e^{\theta_{2} \bar{\xi}(x)}}{e^{\theta_{1} \bar{\xi}(x)}-e^{\theta_{2} \bar{\xi}(x)}} \\
\iota_{\xi}(x)= & \frac{\mathbb{W}_{q}^{\prime}(\bar{\xi}(x))}{\mathbb{W}_{q}(\bar{\xi}(x))} Z_{q}(\bar{\xi}(x))-q \mathbb{W}_{q}(\bar{\xi}(x)) \\
= & \frac{q}{\theta_{1}} \mathbb{W}_{q}^{\prime}(\bar{\xi}(x))+\frac{\mathbb{W}_{q}^{\prime}(\bar{\xi}(x))}{\mathbb{W}_{q}(\bar{\xi}(x))} e^{\theta_{2} \bar{\xi}(x)}-q \mathbb{W}_{q}(\bar{\xi}(x)) \\
= & \frac{q}{\theta_{1}} \kappa\left(\theta_{1} e^{\theta_{1} \bar{\xi}(x)}-\theta_{2} e^{\theta_{2} \bar{\xi}(x)}\right)+\frac{\mathbb{W}_{q}^{\prime}(\bar{\xi}(x))}{\mathbb{W}_{q}(\bar{\xi}(x))} e^{\theta_{2} \bar{\xi}(x)} \\
& -q \kappa\left(e^{\theta_{1} \bar{\xi}(x)}-e^{\theta_{2} \bar{\xi}(x)}\right) \\
= & \frac{\left(\theta_{1}-\theta_{2}\right) e^{\left(\theta_{1}+\theta_{2}\right) \bar{\xi}(x)}}{e^{\theta_{1} \bar{\xi}(x)}-e^{\theta_{2} \bar{\xi}(x)}} .
\end{aligned}
$$

Similarly, it can be verified that

$$
\begin{aligned}
& \widetilde{\varrho}_{\xi}(x)=\frac{\mathbb{W}_{q}^{\prime}(\bar{\xi}(x))}{\mathbb{W}_{q}(\bar{\xi}(x))}=\frac{\widetilde{\theta}_{1} e^{\widetilde{\theta}_{1} \bar{\xi}(x)}-\widetilde{\theta}_{2} e^{\tilde{\theta}_{2} \bar{\xi}(x)}}{e^{\widetilde{\theta}_{1} \bar{\xi}(x)}-e^{\tilde{\theta}_{2} \bar{\xi}(x)}}, \\
& \tilde{\iota}_{\xi}(x)=\frac{\mathbb{W}_{q}^{\prime}(\bar{\xi}(x))}{\mathbb{W}_{q}(\bar{\xi}(x))} Z_{q}(\bar{\xi}(x))-q \mathbb{W}_{q}(\bar{\xi}(x)) \\
& =\frac{\left(\widetilde{\theta}_{1}-\tilde{\theta}_{2}\right) e^{\left(\tilde{\theta}_{1}+\tilde{\theta}_{2}\right) \bar{\xi}(x)}}{e^{\tilde{\theta}_{1}} \bar{\xi}(x)-e^{\tilde{\theta}_{2}} \bar{\xi}(x)} \text {. }
\end{aligned}
$$

Combining (36)-(39), for $x \in[b, \infty)$,

$$
\begin{aligned}
& V_{\xi}(x)=\frac{c_{1}+c_{2}}{q}\left[1-\int_{x}^{\infty} \exp \left(-\int_{x}^{z} \widetilde{\varrho}_{\xi}(w) \mathrm{d} w\right) \tilde{\iota}_{\xi}(z) \mathrm{d} z\right]
\end{aligned}
$$

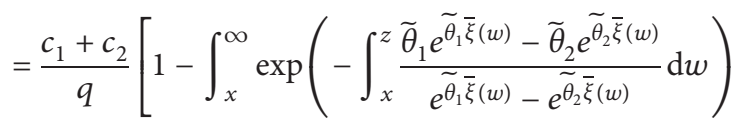

$$
\begin{aligned}
& \left.\frac{\left(\widetilde{\theta}_{1}-\widetilde{\theta}_{2}\right) e^{\left(\widetilde{\theta}_{1}+\tilde{\theta}_{2}\right) \bar{\xi}(z)}}{e^{\tilde{\theta}_{1}} \bar{\xi}(z)-e^{\tilde{\theta}_{2}} \bar{\xi}(z)} \mathrm{d} z\right]
\end{aligned}
$$

and for $x \in[0, b)$,

$$
\begin{aligned}
V_{\xi}(x)= & \frac{c_{1}}{q}-\frac{c_{1}}{q} \int_{x}^{b} \exp \left(-\int_{x}^{z} \varrho_{\xi}(w) \mathrm{d} w\right) \iota_{\xi}(z) \mathrm{d} z+\exp \left(-\int_{x}^{b} \varrho_{\xi}(z) \mathrm{d} z\right)\left(V_{\xi}(b)-\frac{c_{1}}{q}\right) \\
= & \frac{c_{1}}{q}\left[1-\int_{x}^{b} \exp \left(-\int_{x}^{z} \frac{\theta_{1} e^{\theta_{1} \bar{\xi}(w)}-\theta_{2} e^{\theta_{2} \bar{\xi}(w)}}{e^{\theta_{1} \bar{\xi}(w)}-e^{\theta_{2} \bar{\xi}(w)}} \mathrm{d} w\right) \frac{\left(\theta_{1}-\theta_{2}\right) e^{\left(\theta_{1}+\theta_{2}\right) \bar{\xi}(z)}}{e^{\theta_{1} \bar{\xi}(z)}-e^{\theta_{2} \bar{\xi}(z)}} \mathrm{d} z\right] \\
& +\exp \left(-\int_{x}^{b} \frac{\theta_{1} e^{\theta_{1} \bar{\xi}(z)}-\theta_{2} e^{\theta_{2} \bar{\xi}(z)}}{e^{\theta_{1} \bar{\xi}(z)}-e^{\theta_{2} \bar{\xi}(z)}} \mathrm{d} z\right)\left(V_{\xi}(b)-\frac{c_{1}}{q}\right) .
\end{aligned}
$$

Now, we give some numerical illustrations. Consider the following three draw-down functions: $\xi(x)=0, \xi(x)=$ $0.2 x-0.2$, and $\xi(x)=0.5 x-0.2$. As discussed in Remark 1 , when $\xi=0, \tau_{\xi}$ reduces to the classical ruin time, then case (a) can be regarded as the dividend problem until the classical ruin time. For the linear draw-down function $\xi(x)=0.5 x-0.2$, we have that $X_{t}<\xi\left(\bar{X}_{t}\right)=0.5 \bar{X}_{t}-0.2$ at the first draw-down time, which is equivalent to $0.5 \bar{X}_{t}-X_{t}>0.2$. This refers to the first time that the surplus process drops 0.2 units below $50 \%$ of its maximum to date. In practice, the risk manager can use it as a turning point of taking some actions, such as increasing the premium rate $\mu$ to avoid possible disasters.

We choose the parameters of the surplus process as follows: $\mu=2, \sigma=5$ and $q=0.01$. For the values of $c_{1}$ and $c_{2}$ of the ratchet dividend strategy, we consider the following three cases: (a) $c_{1}=0, c_{2}=0.5$, (b) $c_{1}=0.1, c_{2}=0.5$, and (c) $c_{1}=0.1, c_{2}=1$. In Figure 1 , for a fixed initial value $x=1$, we depict the behaviors of $V_{\xi}$ as a function of $b$ for these three cases. Firstly, it is obvious to see the trends in Figure 1(a) that $V_{\xi}$ is a decreasing function of $b$. Note that, in the case $c_{1}=0$, there is no dividend to be paid at the beginning of the surplus process (assume the threshold level $b>x=1$ ); therefore, when the threshold $b$ becomes larger, the surplus process has fewer chances of going above $b$, so the total expected dividend decreases. Secondly, in Figures 1(b) and 1(c), we can see that when $b>1, V_{\xi}$ first increases and then decreases in $b$, namely, $V_{\xi}$ is a concave function of $b$. This phenomenon can be explained by the following two aspects. On the one hand, a smaller value of $b$ means that more dividends may be paid in finite time, but the draw-down (ruin) time may come earlier due to the lower surplus of the process. On the other hand, when the value of $b$ is larger, the surplus process is more difficult to exceed the threshold level $b$ so as to obtain a larger dividend rate, and hence the total expected dividend until the draw-down (ruin) time may be less. The concavity of $V_{\xi}$ in Figures 1(b) and 1(c) motivates us to find the optimal threshold (denote it by $b^{*}$ ). By (26), we can get the corresponding optimal threshold level $b^{*}$ for different cases, as shown in Table 1.

Example 2. Let $Y_{t}=x+c t-\sum_{i=1}^{N_{t}} Z_{i}, t \geq 0$, where $N=$ $\left\{N_{t}\right\}_{t \geq 0}$ is a Poisson process with intensity $\lambda>0$ and $Z_{i}$ are i.i.d. exponential random variables with parameter $\mu$. In 


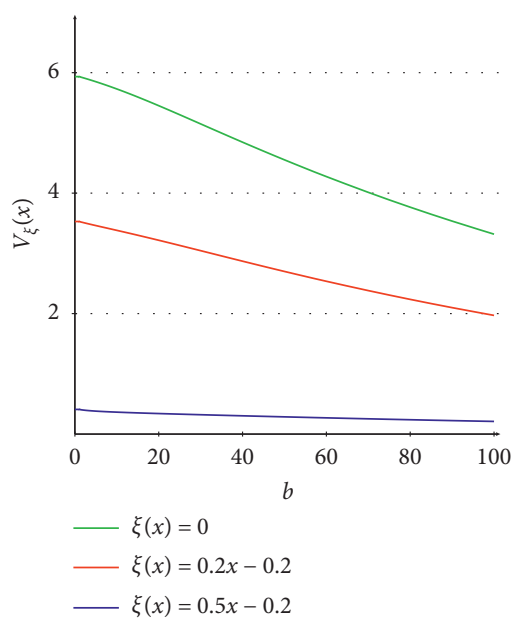

(a)

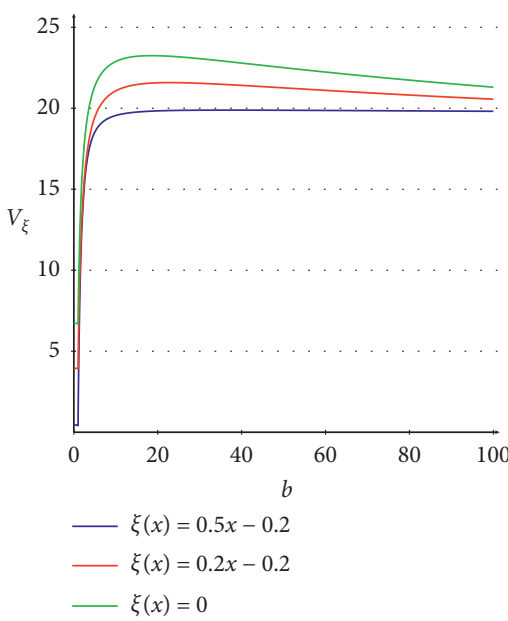

(b)

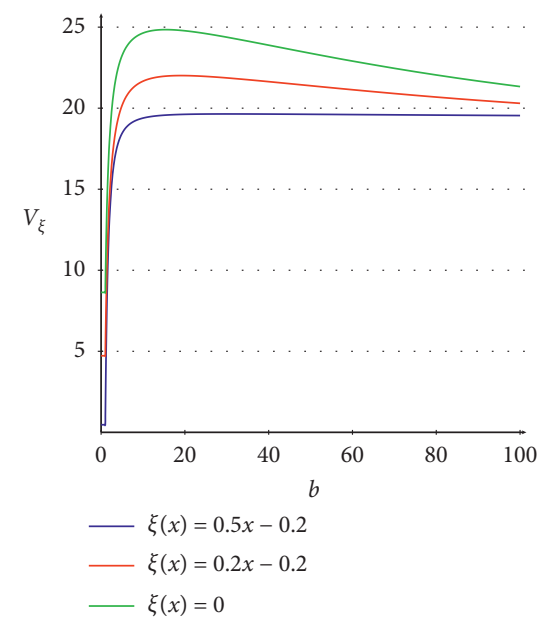

(c)

Figure 1: Plot of $V_{\xi}$ as a function of $b$ for the Brownian motion with drift with $q=0.01, \sigma=5$. (a) $c_{1}=0, c_{2}=0.5, c=2,(\mathrm{~b}) c_{1}=0.1, c_{2}=0.5$, $\mu=2$, and (c) $c_{1}=0.1, c_{2}=1, \mu=2$.

TABLE 1: Exact values of $b^{*}$ for the Brownian motion with drift.

\begin{tabular}{lccc}
\hline The optimal $b^{*}$ & $\xi(x)=0$ & $\xi(x)=0.2 x-0.2$ & $\xi(x)=0.5 x-0.2$ \\
\hline$c_{1}=0.1, c_{2}=0.5$ & 18.53 & 22.92 & 36.70 \\
$c_{1}=0.1, c_{2}=1$ & 15.46 & 19.15 & 30.26 \\
\hline
\end{tabular}

addition, let $X_{t}=x+\left(c-c_{1}\right) t-\sum_{i=1}^{N_{t}} Z_{i}, t \geq 0$ and $\tilde{X}_{t}=x+$ $\left(c-c_{1}-c_{2}\right) t-\sum_{i=1}^{N_{t}} Z_{i}, t \geq 0$. The scale functions associated with $X$ and $\widetilde{X}$ are given, respectively, by

$$
\begin{aligned}
& \mathbb{W}_{q}(x)=\frac{A_{1}}{c-c_{1}} e^{\theta_{1} x}-\frac{A_{2}}{c-c_{1}} e^{\theta_{2} x}, \\
& \mathbb{W}_{q}(x)=\frac{\tilde{A}_{1}}{c-c_{1}-c_{2}} e^{\tilde{\theta}_{1} x}-\frac{\tilde{A}_{2}}{c-c_{1}-c_{2}} e^{\tilde{\theta}_{2} x},
\end{aligned}
$$

where

$$
\begin{aligned}
A_{1} & =\frac{\mu+\theta_{1}}{\theta_{1}-\theta_{2}}, \\
A_{2} & =\frac{\mu+\theta_{2}}{\theta_{1}-\theta_{2}}, \\
\theta_{1} & =\frac{\lambda+q-\left(c-c_{1}\right) \mu+K}{2\left(c-c_{1}\right)}, \\
\theta_{2} & =\frac{\lambda+q-\left(c-c_{1}\right) \mu-K}{2\left(c-c_{1}\right)}, \\
K & =\sqrt{\left(\left(c-c_{1}\right) \mu-\lambda-q\right)^{2}+4\left(c-c_{1}\right) q \mu}, \\
\widetilde{A}_{1} & =\frac{\mu+\tilde{\theta}_{1}}{\widetilde{\theta}_{1}-\widetilde{\theta}_{2}} \\
\widetilde{A}_{2} & =\frac{\mu+\widetilde{\theta}_{2}}{\tilde{\theta}_{1}-\widetilde{\theta}_{2}}
\end{aligned}
$$

$$
\begin{aligned}
\tilde{\theta}_{1} & =\frac{\lambda+q-\left(c-c_{1}-c_{2}\right) \mu+\widetilde{K}}{2\left(c-c_{1}-c_{2}\right)}, \\
\tilde{\theta}_{2} & =\frac{\lambda+q-\left(c-c_{1}-c_{2}\right) \mu-\widetilde{K}}{2\left(c-c_{1}-c_{2}\right)}, \\
\widetilde{K} & =\sqrt{\left(\left(c-c_{1}-c_{2}\right) \mu-\lambda-q\right)^{2}+4\left(c-c_{1}-c_{2}\right) q \mu} . \\
\widetilde{A}_{1} & =\frac{\mu+\tilde{\theta}_{1}}{\tilde{\theta}_{1}-\widetilde{\theta}_{2}}, \\
\widetilde{A}_{2} & =\frac{\mu+\tilde{\theta}_{2}}{\widetilde{\theta}_{1}-\widetilde{\theta}_{2}}, \\
\tilde{\theta}_{1} & =\frac{\lambda+q-\left(c-c_{1}-c_{2}\right) \mu+\widetilde{K}}{2\left(c-c_{1}-c_{2}\right)}, \\
\tilde{\theta}_{2} & =\frac{\lambda+q-\left(c-c_{1}-c_{2}\right) \mu-\widetilde{K}}{2\left(c-c_{1}-c_{2}\right)}, \\
\widetilde{K} & =\sqrt{\left(\left(c-c_{1}-c_{2}\right) \mu-\lambda-q\right)^{2}+4\left(c-c_{1}-c_{2}\right) q \mu} .
\end{aligned}
$$

From (42), we have

$$
\begin{aligned}
Z_{q}(x)= & +\frac{q A_{1}}{\left(c-c_{1}\right) \theta_{1}}\left(e^{\theta_{1} x}-1\right) \\
& -\frac{q A_{2}}{\left(c-c_{1}\right) \theta_{2}}\left(e^{\theta_{2} x}-1\right), \quad \text { for } x, q \geq 0, \\
\mathbb{Z}_{q}(x)=1 & +\frac{q \widetilde{A}_{1}}{\left(c-c_{1}-c_{2}\right) \widetilde{\theta}_{1}}\left(e^{\tilde{\theta}_{1} x}-1\right) \\
& -\frac{q \widetilde{A}_{2}}{\left(c-c_{1}-c_{2}\right) \widetilde{\theta}_{2}}\left(e^{\tilde{\theta}_{2} x}-1\right), \quad \text { for } x, q \geq 0 .
\end{aligned}
$$

It follows from (42) and (44) that 


$$
\begin{aligned}
\varrho_{\xi}(x)= & \frac{\mathbb{W}_{q}^{\prime}(\bar{\xi}(x))}{\mathbb{W}_{q}(\bar{\xi}(x))}=\frac{A_{1} \theta_{1} e^{\theta_{1} \bar{\xi}(x)}-A_{2} \theta_{2} e^{\theta_{2} \bar{\xi}(x)}}{A_{1} e^{\theta_{1} \bar{\xi}(x)}-A_{2} e^{\theta_{2} \bar{\xi}(x)}}, \\
\iota_{\xi}(x)= & \frac{\mathbb{W}_{q}^{\prime}(\bar{\xi}(x))}{\mathbb{W}_{q}(\bar{\xi}(x))} Z_{q}(\bar{\xi}(x))-q \mathbb{W}_{q}(\bar{\xi}(x)) \\
= & \frac{A_{1} \theta_{1} e^{\theta_{1} \bar{\xi}(x)}-A_{2} \theta_{2} e^{\theta_{2} \bar{\xi}(x)}}{A_{1} e^{\theta_{1} \bar{\xi}(x)}-A_{2} e^{\theta_{2} \bar{\xi}(x)}} \\
= & \left.\frac{q A_{1}}{A_{1} e^{\theta_{1} \bar{\xi}(x)}-A_{2} e^{\theta_{2} \bar{\xi}(x)}}\left(e^{\theta_{1} \bar{\xi}(x)}-1\right)-\frac{q A_{2}}{\left(c-c_{1}\right) \theta_{2}}\left(e^{\theta_{2} \bar{\xi}(x)}-1\right)\right] \\
& {\left[\left(1-\frac{q A_{1}}{\left(c-c_{1}\right) \theta_{1}}+\frac{q A_{2}}{\left(c-c_{1}\right) \theta_{2}}\right) A_{1} \theta_{1} e^{\theta_{1} \bar{\xi}(x)}\right.} \\
& -\left(1-\frac{q A_{1}}{\left(c-c_{1}\right) \theta_{1}}+\frac{q A_{2}}{\left(c-c_{1}\right) \theta_{2}}\right) A_{2} \theta_{2} e^{\theta_{2} \bar{\xi}(x)} \\
& \left.+\frac{q A_{1} A_{2}}{\left(c-c_{1}\right)}\left(2-\frac{\theta_{1}}{\theta_{2}}-\frac{\theta_{2}}{\theta_{1}}\right) e^{\left(\theta_{1}+\theta_{2}\right) \bar{\xi}(x)}\right] .
\end{aligned}
$$

In addition, plugging (42) and (45) into (24) and (25) leads to

$$
\begin{aligned}
& \widetilde{\varrho}_{\xi}(x)=\frac{\widetilde{A}_{1} \widetilde{\theta}_{1} e^{\tilde{\theta}_{1} \bar{\xi}(x)}-\widetilde{A}_{2} \widetilde{\theta}_{2} e^{\tilde{\theta}_{2} \bar{\xi}(x)}}{\widetilde{A}_{1} e^{\tilde{\theta}_{1} \bar{\xi}(x)}-\widetilde{A}_{2} e^{\tilde{\theta}_{2} \bar{\xi}(x)}}, \\
& \tau_{\xi}(x)=\frac{1}{\widetilde{A}_{1} e^{\widetilde{\theta}_{1} \bar{\xi}(x)}-\widetilde{A}_{2} e^{\widetilde{\theta}_{2} \bar{\xi}(x)}}\left[\left(1-\frac{q \widetilde{A}_{1}}{\left(c-c_{1}-c_{2}\right) \widetilde{\theta}_{1}}\right.\right. \\
& \left.+\frac{q \widetilde{A}_{2}}{\left(c-c_{1}-c_{2}\right) \widetilde{\theta}_{2}}\right) \widetilde{A}_{1} \widetilde{\theta}_{1} e^{\tilde{\theta}_{1} \bar{\xi}(x)}-\left(1-\frac{q \widetilde{A}_{1}}{\left(c-c_{1}-c_{2}\right) \widetilde{\theta}_{1}}\right. \\
& \left.+\frac{q \widetilde{A}_{2}}{\left(c-c_{1}-c_{2}\right) \widetilde{\theta}_{2}}\right) \widetilde{A}_{2} \widetilde{\theta}_{2} e^{\tilde{\theta}_{2} \bar{\xi}(x)}+\frac{q \widetilde{A}_{1} \widetilde{A}_{2}}{\left(c-c_{1}-c_{2}\right)} \\
& \left.\left(2-\frac{\tilde{\theta}_{1}}{\tilde{\theta}_{2}}-\frac{\tilde{\theta}_{2}}{\tilde{\theta}_{1}}\right) e^{\left(\tilde{\theta}_{1}+\tilde{\theta}_{2}\right) \bar{\xi}(x)}\right] \text {. }
\end{aligned}
$$

Combining (13), (14), and (46)-(49) yields

$$
\begin{aligned}
& V_{\xi}(x)=\frac{c_{1}+c_{2}}{q}\left(1-\int_{x}^{\infty} \exp \left(-\int_{x}^{z} \tilde{\varrho}_{\xi}(w) \mathrm{d} w\right) \tilde{l}_{\xi}(z) \mathrm{d} z\right) \\
& =\frac{c_{1}+c_{2}}{q}\left\{1-\int_{x}^{\infty} \exp \left(-\int_{x}^{z} \frac{\widetilde{A}_{1} \widetilde{\theta}_{1} e^{\theta_{1} \bar{\xi}(w)}-\widetilde{A}_{2} \widetilde{\theta}_{2} e^{\tilde{\theta}_{2} \bar{\xi}(w)}}{\widetilde{A}_{1} e^{\widetilde{\theta}_{1} \bar{\xi}(w)}-\widetilde{A}_{2} \tilde{e}^{\widetilde{\theta}_{2}} \bar{\xi}(w)} \mathrm{d} w\right) \frac{1}{\widetilde{A}_{1} e^{\widetilde{\theta}_{1} \bar{\xi}(z)}-\widetilde{A}_{2} e^{\tilde{\theta}_{2} \bar{\xi}(z)}}\right. \\
& \times\left[\left(1-\frac{q \tilde{A}_{1}}{\left(c-c_{1}-c_{2}\right) \widetilde{\theta}_{1}}+\frac{q \tilde{A}_{2}}{\left(c-c_{1}-c_{2}\right) \tilde{\theta}_{2}}\right) \widetilde{A}_{1} \widetilde{\theta}_{1} e^{\tilde{\theta}_{1} \bar{\xi}(z)}-\left(1-\frac{q \widetilde{A}_{1}}{\left(c-c_{1}-c_{2}\right) \widetilde{\theta}_{1}}+\frac{q \widetilde{A}_{2}}{\left(c-c_{1}-c_{2}\right) \widetilde{\theta}_{2}}\right) \tilde{A}_{2} \widetilde{\theta}_{2} e^{\tilde{\theta}_{2} \bar{\xi}(z)}\right. \\
& \left.\left.+\frac{q \widetilde{A}_{1} \widetilde{A}_{2}}{\left(c-c_{1}-c_{2}\right)}\left(2-\frac{\tilde{\theta}_{1}}{\tilde{\theta}_{2}}-\frac{\tilde{\theta}_{2}}{\tilde{\theta}_{1}}\right) e^{\left(\tilde{\theta}_{1}+\tilde{\theta}_{2}\right) \bar{\xi}(z)}\right] \mathrm{d} z\right\}, \quad x \in[b, \infty), \\
& V_{\xi}(x)=\frac{c_{1}}{q}-\frac{c_{1}}{q} \int_{x}^{b} \exp \left(-\int_{x}^{z} \varrho_{\xi}(w) \mathrm{d} w\right) \iota_{\xi}(z) \mathrm{d} z+\exp \left(-\int_{x}^{b} \varrho_{\xi}(z) \mathrm{d} z\right)\left(V_{\xi}(b)-\frac{c_{1}}{q}\right) \\
& =\frac{c_{1}}{q}\left\{1-\int_{x}^{b} \exp \left(-\int_{x}^{z} \frac{A_{1} \theta_{1} e^{\theta_{1} \bar{\xi}(w)}-A_{2} \theta_{2} e^{\theta_{2} \bar{\xi}(w)}}{A_{1} e^{\theta_{1} \bar{\xi}(w)}-A_{2} e^{\theta_{2} \bar{\xi}(w)}} \mathrm{d} w\right) \frac{1}{A_{1} e^{\theta_{1} \bar{\xi}(z)}-A_{2} e^{\theta_{2} \bar{\xi}(z)}}\right\} \\
& {\left[\left(1-\frac{q A_{1}}{\left(c-c_{1}\right) \theta_{1}}+\frac{q A_{2}}{\left(c-c_{1}\right) \theta_{2}}\right) A_{1} \theta_{1} e^{\theta_{1} \bar{\xi}(z)}-\left(1-\frac{q A_{1}}{\left(c-c_{1}\right) \theta_{1}}+\frac{q A_{2}}{\left(c-c_{1}\right) \theta_{2}}\right) \times A_{2} \theta_{2} e^{\theta_{2} \bar{\xi}(z)}\right.} \\
& \left.\left.+\frac{q A_{1} A_{2}}{\left(c-c_{1}\right)}\left(2-\frac{\theta_{1}}{\theta_{2}}-\frac{\theta_{2}}{\theta_{1}}\right) e^{\left(\theta_{1}+\theta_{2}\right) \bar{\xi}(z)}\right] \mathrm{d} z\right\} \\
& +\exp \left(-\int_{x}^{b} \frac{A_{1} \theta_{1} e^{\theta_{1} \bar{\xi}(z)}-A_{2} \theta_{2} e^{\theta_{2} \bar{\xi}(z)}}{A_{1} e^{\theta_{1} \bar{\xi}(z)}-A_{2} e^{\theta_{2} \bar{\xi}(z)}} \mathrm{d} z\right)\left(V_{\xi}(b)-\frac{c_{1}}{q}\right), \quad x \in[0, b) \text {. }
\end{aligned}
$$

Now, we give some numerical illustrations for this example. We still consider these three draw-down functions as given in Example 1: $\xi(x)=0, \xi(x)=0.2 x-0.2$, and $\xi(x)=0.5 x-0.2$. We choose the parameters of the surplus process as follows: $c=2, \lambda=\mu=1$ and $q=0.01$. For the values of $c_{1}$ and $c_{2}$ of the ratchet dividend strategy we also consider the following three cases: (a) $c_{1}=0, c_{2}=0.5$, (b) $c_{1}=0.1$, $c_{2}=0.5$, and (c) $c_{1}=0.1, c_{2}=1$. In Figures $2(\mathrm{a})-2(\mathrm{c})$, for $\mathrm{a}$ 


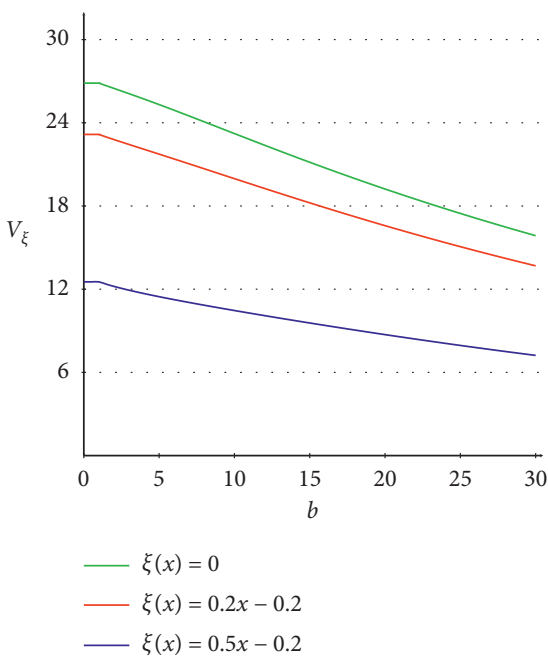

(a)

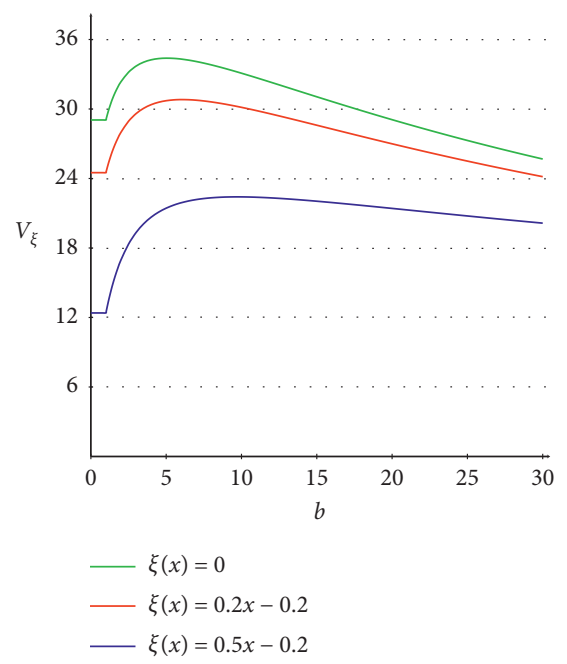

(b)

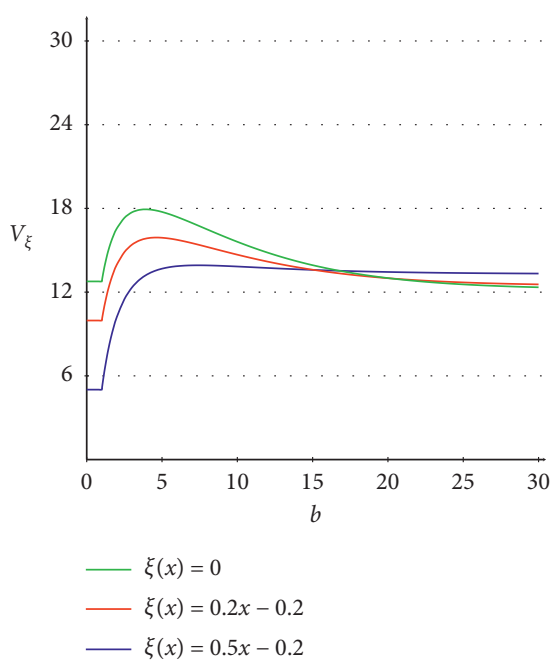

(c)

Figure 2: Plot of $V_{\xi}$ as a function of $b$ for the compound Poisson process with $\lambda=\mu=1, q=0.01$. (a) $c_{1}=0, c_{2}=0.5, c=2$, (b) $c_{1}=0.1, c_{2}=0.5$, $c=2$, and (c) $c_{1}=0.1, c_{2}=1, c=2$.

TABLe 2: Exact values of $b^{*}$ for the compound Poisson model.

\begin{tabular}{lccc}
\hline The optimal $b^{*}$ & $\xi(x)=0$ & $\xi(x)=0.2 x-0.2$ & $\xi(x)=0.5 x-0.2$ \\
\hline$c_{1}=0.1, c_{2}=0.5$ & 5.06 & 6.09 & 9.72 \\
$c_{1}=0.1, c_{2}=1$ & 3.90 & 4.63 & 7.41
\end{tabular}

fixed initial value $x=1$, we depict the behaviors of $V_{\xi}$ as a function of $b$ for these three cases. The trends of $V_{\xi}$ can be similarly explained, as in Example 1. Furthermore, by (26), we can also get the corresponding optimal threshold level $b^{*}$ for the compound Poisson risk model, which is shown in Table 2.

\section{Data Availability}

The data used to support the results of this study are included within the supplementary information files.

\section{Conflicts of Interest}

The authors declare that they have no conflicts of interest.

\section{Acknowledgments}

This work was supported by the Science and Technology Planning Project of Jiangxi Provincial Education Department (no. GJJ180201).

\section{Supplementary Materials}

(1) Attachments 1 and 2 include the Matlab operation programs of case 1 (in Section 4: numerical illustrations for $V_{\xi}$ (as a function of $b$ ) and for solving the optimal threshold level $b^{*}$ (for the Brownian motion with drift) of this manuscript). (2) Attachments 3 and 4 include the Matlab operation programs of case 2 (in Section 4: numerical illustrations for $V_{\xi}$ (as a function of $b$ ) and for solving the optimal threshold level $b^{*}$ (for the compound Poison process) of this manuscript). (Supplementary Materials)

\section{References}

[1] B. De Finetti, "Su unimpostazione alternativa della teoria collettiva del rischio," Transactions of the XVth International Congress of Actuaries, vol. 2, no. 1, pp. 433-443, 1957.

[2] M. H. Miller and F. Modigliani, "Dividend policy, growth, and the valuation of shares," The Journal of Business, vol. 34 , no. 4 , pp. 411-433, 1961.

[3] R. L. Loeffen, "On optimality of the barrier strategy in de Finetti's dividend problem for spectrally negative lévy processes," The Annals of Applied Probability, vol. 18, no. 5, pp. 1669-1680, 2008.

[4] R. L. Loeffen and J.-F. Renaud, "De Finetti's optimal dividends problem with an affine penalty function at ruin," Insurance: Mathematics and Economics, vol. 46, no. 1, pp. 98-108, 2010.

[5] C. Yin and C. Wang, "Optimality of the barrier strategy in de Finetti's dividend problem for spectrally negative lévy processes: an alternative approach," Journal of Computational and Applied Mathematics, vol. 233, no. 2, pp. 482-491, 2009.

[6] K. C. Yuen and C. Yin, "On optimality of the barrier strategy for a general Lévy risk process," Mathematical and Computer Modelling, vol. 53, no. 9-10, pp. 1700-1707, 2011.

[7] W. Wang and X. Zhou, "General drawdown-based de Finetti optimization for spectrally negative lévy risk processes," Journal of Applied Probability, vol. 55, no. 2, pp. 513-542, 2018.

[8] W. Yu, F. Wang, Y. Huang, and H. Liu, "Social optimal mean field control problem for population growth model," Asian Journal of Control, pp. 1-8, 2019.

[9] H. U. Gerber and E. S. W. Shiu, "On optimal dividends: from reflection to refraction," Journal of Computational and Applied Mathematics, vol. 186, no. 1, pp. 4-22, 2006.

[10] M. Jeanblanc-Picqué and A. N. Shiryaev, "Optimization of the flow of dividends," Uspekhi Matematicheskikh Nauk, vol. 50, no. 2, pp. 25-46, 1995. 
[11] S. Asmussen and M. Taksar, "Controlled diffusion models for optimal dividend pay-out," Insurance: Mathematics and Economics, vol. 20, no. 1, pp. 1-15, 1997.

[12] H. U. Gerber and E. S. W. Shiu, "On optimal dividend strategies in the compound Poisson model," North American Actuarial Journal, vol. 10, no. 2, pp. 76-93, 2006.

[13] X. Peng, W. Su, and Z. Zhang, "On a perturbed compound Poisson risk model under a periodic threshold-type dividend strategy," Journal of Industrial \& Management Optimization, vol. 13, no. 5, pp. 1-20, 2017.

[14] Z. Liu, P. Chen, and Y. Hu, "On the dual risk model with diffusion under a mixed dividend strategy," Applied Mathematics and Computation, vol. 376, Article ID 125115, 2020.

[15] B. Avanzi, J.-L. Pérez, B. Wong, and K. Yamazaki, "On optimal joint reflective and refractive dividend strategies in spectrally positive lévy models," Insurance: Mathematics and Economics, vol. 72, pp. 148-162, 2017.

[16] H. Albrecher, N. Bäuerle, and M. Bladt, "Dividends: from refracting to ratcheting," Insurance: Mathematics and Economics, vol. 83, pp. 47-58, 2018.

[17] F. Avram, N. L. Vu, and X. Zhou, "On taxed spectrally negative lévy processes with draw-down stopping," Insurance: Mathematics and Economics, vol. 76, pp. 69-74, 2017.

[18] H. Albrecher and S. Thonhauser, "Optimality results for dividend problems in insurance. RACSAM-revista de la real academia de ciencias exactas, fisicasy naturales," Revista de la Real Academia de Ciencias Exactas, Fisicas y Naturales. Serie A. Matematicas, vol. 103, no. 2, pp. 295-320, 2009.

[19] W. Wang and X. Zhou, "A drawdown reflected spectrally negative lévy process," Journal of Theoretical Probability, pp. 1-24, 2019.

[20] W. Wang and X. Zhou, "Draw-down parisian ruin for spectrally negative lévy process," 2019, https://arxiv.org/abs/ 1904.03286.

[21] I. Czarna and Z. Palmowski, "Dividend problem with parisian delay for a spectrally negative lévy risk process," Journal of Optimization Theory and Applications, vol. 161, no. 1, pp. 239-256, 2014.

[22] W. Wang and Z. Zhang, "Optimal loss-carry-forward taxation for lévy risk processes stopped at general draw-down time," Advances in Applied Probability, vol. 51, no. 3, pp. 865-897, 2019.

[23] W. Wang and R. Ming, "Two-side exit problems for taxed lévy risk process involving the general draw-down time," Statistics \& Probability Letters, vol. 138, pp. 66-74, 2018.

[24] W. Wang, P. Chen, and S. Li, "Generalized expected discounted penalty function at general drawdown for lévy risk processes. Insurance: mathematics and economics," 2020, https://arxiv.org/abs/1906.01449.

[25] X. Chen, D. Landriault, B. Li, and D. Li, "On minimizing drawdown risks of lifetime investments," Insurance: Mathematics and Economics, vol. 65, pp. 46-54, 2015.

[26] J. Ruan, W. Yu, K. Song, Y. Sun, Y. Huang, and X. Yu, “A note on a generalized Gerber-Shiu discounted penalty function for a compound Poisson risk model," Mathematics, vol. 7, no. 10, p. 891, 2019.

[27] W. Yu, Y. Yong, G. Guan, Y. Huang, W. Su, and C. Cui, "Valuing guaranteed minimum death benefits by cosine series expansion," Mathematics, vol. 7, no. 9, p. 835, 2019.

[28] Z. Zhang, Y. Yong, and W. Yu, "Valuing equity-linked death benefits in general exponential lévy models," Journal of Computational and Applied Mathematics, vol. 365, Article ID 112377, 2020.
[29] A. Kuznetsov, A. E. Kyprianou, and V. Rivero, "The theory of scale functions for spectrally negative Lévy processes," in Lévy matters II, pp. 97-186, Springer, Berlin, Germany, 2012.

[30] D. Landriault, B. Li, and H. Zhang, "On magnitude, asymptotics and duration of drawdowns for Lévy models," Bernoulli, vol. 23, no. 1, pp. 432-458, 2017.

[31] B. Li, N. L. Vu, and X. Zhou, "Exit problems for general drawdown times of spectrally negative lévy processes," Journal of Applied Probability, vol. 56, no. 2, pp. 441-457, 2019. 